\title{
Constraining the recent star formation history of galaxies: an approximate Bayesian computation approach
}

\author{
G. Aufort ${ }^{1,2}$, L. Ciesla ${ }^{2}$, P. Pudlo ${ }^{1}$, and V. Buat ${ }^{3}$ \\ 1 Aix Marseille Université, CNRS, Centrale Marseille, I2M, Marseille, France \\ e-mail: gregoire. aufort@univ-amu.fr \\ 2 Aix-Marseille Université, CNRS, LAM (Laboratoire d'Astrophysique de Marseille) UMR7326, 13388 Marseille, France \\ 3 Aix-Marseille Université, CNRS, LAM (Laboratoire d'Astrophysique de Marseille) UMR7326, Institut Universitaire de France \\ (IUF), 13388 Marseille, France
}

Received 26 September 2019 / Accepted 12 February 2020

\begin{abstract}
Although galaxies are found to follow a tight relation between their star formation rate and stellar mass, they are expected to exhibit complex star formation histories (SFH) with short-term fluctuations. The goal of this pilot study is to present a method that identifies galaxies that undergo strong variation in star formation activity in the last ten to some hundred million years. In other words, the proposed method determines whether a variation in the last few hundred million years of the SFH is needed to properly model the spectral energy distribution (SED) rather than a smooth normal SFH. To do so, we analyzed a sample of COSMOS galaxies with $0.5<z<1$ and $\log M_{*}>8.5$ using high signal-to-noise ratio broadband photometry. We applied approximate Bayesian computation, a custom statistical method for performing model choice, which is associated with machine-learning algorithms to provide the probability that a flexible SFH is preferred based on the observed flux density ratios of galaxies. We present the method and test it on a sample of simulated SEDs. The input information fed to the algorithm is a set of broadband UV to NIR (rest-frame) flux ratios for each galaxy. The choice of using colors is made to remove any difficulty linked to normalization when classification algorithms are used. The method has an error rate of $21 \%$ in recovering the correct SFH and is sensitive to SFR variations larger than 1 dex. A more traditional SED-fitting method using CIGALE is tested to achieve the same goal, based on fit comparisons through the Bayesian information criterion, but the best error rate we obtained is higher, $28 \%$. We applied our new method to the COSMOS galaxies sample. The stellar mass distribution of galaxies with a strong to decisive evidence against the smooth delayed- $\tau$ SFH peaks at lower $M_{*}$ than for galaxies where the smooth delayed- $\tau$ SFH is preferred. We discuss the fact that this result does not come from any bias due to our training. Finally, we argue that flexible SFHs are needed to be able to cover the largest possible SFR- $M_{*}$ parameter space.
\end{abstract}

Key words. galaxies: evolution - galaxies: fundamental parameters

\section{Introduction}

The tight relation linking the star formation rate (SFR) and stellar mass of star-forming galaxies, the so-called main sequence (MS), opened a new window in our understanding of galaxy evolution (Elbaz et al. 2007; Noeske et al. 2007). It implies that the majority of galaxies are likely to form the bulk of their stars through steady-state processes rather than violent episodes of star formation. However, this relation has a scatter of $\sim 0.3 \mathrm{dex}$ (Schreiber et al. 2015) that is found to be relatively constant at all masses and over cosmic time (Guo et al. 2013; Ilbert et al. 2015; Schreiber et al. 2015). One possible explanation of this scatter could be its artificial creation by the accumulation of errors in the extraction of photometric measurements and/or in the determination of the SFR and stellar mass in relation with model uncertainties. However, several studies have found a coherent variation in physical galaxy properties such as the gas fraction (Magdis et al. 2012), Sérsic index and effective radius (Wuyts et al. 2011), and $U-V$ color (e.g., Salmi et al. 2012), suggesting that the scatter is more strongly related to the physics than to measurement and model uncertainties. Furthermore, oscillations in SFR resulting from a varying infall rate and compaction of star formation have been proposed to explain the MS scatter (Sargent et al. 2014; Scoville et al. 2016; Tacchella et al. 2016) and en even be suggested by some simulations (e.g., Dekel \& Burkert 2014).
To decipher whether the scatter is indeed due to variations in star formation history (SFH), we must be able to place a constraint on the recent SFH of galaxies to reconstruct their path along the MS. This information is embedded in the spectral energy distribution (SED) of galaxies. However, recovering it through SED modeling is complex and subject to many uncertainties and degeneracies. Galaxies are indeed expected to exhibit complex SFHs, with short-term fluctuations. This requires sophisticated SFH parametrizations to model them (e.g., Lee et al. 2010; Pacifici et al. 2013, 2016; Behroozi et al. 2013; Leja et al. 2019). The implementation of these models is complex, and large libraries are needed to model all galaxy properties. Numerous studies have instead used simple analytical forms to model galaxies SFH (e.g., Papovich et al. 2001; Maraston et al. 2010; Pforr et al. 2012; Gladders et al. 2013; Simha et al. 2014; Buat et al. 2014; Boquien et al. 2014; Ciesla et al. 2015, 2016, 2017; Abramson et al. 2016). However, SFH parameters are known to be difficult to constrain from broadband SED modeling (e.g., Maraston et al. 2010; Pforr et al. 2012; Buat et al. 2014; Ciesla et al. 2015, 2017; Carnall et al. 2019).

Ciesla et al. (2016) and Boselli et al. (2016) have shown in a sample of well-known local galaxies benefiting from a wealth of ancillary data that a drastic and recent decrease in star formation activity of galaxies can be probed as long as a good UV to near-IR (NIR) rest frame coverage is available. They showed that 
the intensity in the variation of the star formation (SF) activity can be relatively well constrained from broadband SED fitting. Spectroscopy is required, however, to bring information on the time when the change in star formation activity occurred (Boselli et al. 2016). These studies were made on well-known sources of the Virgo cluster, for which the quenching mechanism (ram pressure stripping) is known and HI observations allow a direct verification of the SED modeling results. To go a step further, Ciesla et al. (2018) have blindly applied the method on the GOODSSouth sample to identify sources that underwent a recent and drastic decrease in their SF activity. They compared the quality of the results from SED fitting using two different SFHs and obtained a sample of galaxies where a modeled recent and strong decrease in SFR produced significantly better fits of the broadband photometry. In this work, we improve the method of Ciesla et al. (2018) by gaining in power by applying a custom statistical method to a subsample of COSMOS galaxies to perform the SFH choice: the approximate Bayesian computation (ABC, see, e.g., Marin et al. 2012; Sisson et al. 2018). Based on the observed SED of a galaxy, we wish to choose the most appropriate SFH in a finite set. The main idea behind ABC is to rely on many simulated SEDs generated from all the SFHs in competition using parameters drawn from the prior.

The paper is organized as follows: Sect. 2 describes the astrophysical problem and presents the sample. In Sect. 3 we present the statistical approach as well as the results obtained from a catalog of simulated SEDs of COSMOS-like galaxies. In Sect. 4 we compare the results of this new approach with more traditional SED modeling methods, and apply it to real COSMOS galaxies in Sect. 5. Our results are discussed in Sect. 6.

\section{Constraining the recent star formation history of galaxies using broadband photometry}

\subsection{Building upon the method of Ciesla et al. (2018)}

The main purpose of the study presented in Ciesla et al. (2018) was to probe variations in SFH that occurred on very short timescales, that is, within some hundred million years. Largenumber statistics was needed to be able to catch galaxies at the moment when these variations occurred. The authors aimed at identifying galaxies that recently underwent a rapid $(<500 \mathrm{Myr})$ and drastic downfall in SFR (more than $80 \%$ ) from broadband SED modeling because large photometric samples can provide the statistics needed to pinpoint these objects.

To perform their study, they took advantage of the versatility of the SED modeling code CIGALE ${ }^{1}$ (Boquien et al. 2019). CIGALE is a SED modeling software package that has two functions: a modeling function to create SEDs from a set of given parameters, and an SED fitting function to derive the physical properties of galaxies from observations. Galaxy SEDs are computed from UV-to-radio taking into account the balance between the energy absorbed by dust in the UV-NIR and remitted in IR. To build the SEDs, CIGALE uses a combination of modules including the SFH assumption, which may be analytical, stochastic, or outputs from simulations (e.g., Boquien et al. 2014; Ciesla et al. 2015, 2017), the stellar emission from stellar population models (Bruzual \& Charlot 2003; Maraston 2005), the nebular lines, and the attenuation by dust (e.g., Calzetti et al. 2000; Charlot \& Fall 2000).

Ciesla et al. (2018) compared the results of SED fitting in a sample of GOODS-South galaxies using two different SFHs: one normal delayed- $\tau$ SFH, and one flexible SFH that modeled

\footnotetext{
https://cigale.lam.fr/
}

a truncation of the SFH. The normal delayed- $\tau$ SFH is given by the equation

$\operatorname{SFR}(t) \propto t \times \exp \left(-t / \tau_{\text {main }}\right)$,

where SFR is the star formation rate, $t$ is the time, and $\tau_{\text {main }}$ is the e-folding time. Examples of delayed- $\tau$ SFHs are shown in Fig. 1 for different values of $\tau_{\text {main }}$. The flexible SFH is an extension of the delayed- $\tau$ model,

$\operatorname{SFR}(t) \propto\left\{\begin{array}{ll}t \times \exp \left(-t / \tau_{\text {main }}\right), & \text { when } t \leq t_{\text {flex }} \\ r_{\mathrm{SFR}} \times \operatorname{SFR}\left(t=t_{\text {flex }}\right), & \text { when } t>t_{\text {flex }}\end{array}\right.$,

where $t_{\text {flex }}$ is the time at which the star formation is instantaneously affected, and $r_{\mathrm{SFR}}$ is the ratio between $\operatorname{SFR}\left(t>t_{\mathrm{flex}}\right)$ and $\operatorname{SFR}\left(t=t_{\text {flex }}\right)$,

$r_{\mathrm{SFR}}=\frac{\operatorname{SFR}\left(t>t_{\text {flex }}\right)}{\operatorname{SFR}\left(t_{\mathrm{flex}}\right)}$.

A representation of flexible SFHs is also shown in Fig. 1. The normal delayed- $\tau$ SFH is at first order a particular case of the flexible SFH for which $r_{\mathrm{SFR}}=1$.

To distinguish between the two models, Ciesla et al. (2018) estimated the Bayesian information criterion (BIC, see Sect. 3.2) that is linked to the two models and placed conservative limits on the difference between the two BICs to select the bestsuited model. They showed that a handful of sources were better fit using the flexible SFH, which assumes a recent instantaneous break in the SFH, compared to the more commonly used delayed $\tau$ SFH. They discussed that these galaxies have indeed physical properties that are different from the main population and characteristic of sources in transition.

The limited number of sources identified in the study of Ciesla et al. (2018; 102 out of 6680) was due to their choice to be conservative in their approach and find a clean sample of sources that underwent a rapid quenching of star formation. They imposed that the instantaneous decrease of SFR was more than $80 \%$ and that the BIC difference was larger than 10 . These criteria prevent a complete study of rapid variations in the SFH of galaxies because many of them would be missed. Furthermore, only decreases in SFR were considered and not the opposite, that is, star formation bursts. Finally, their method is time consuming because the CIGALE code has to be run twice, once per SFH model considered, to perform the analysis. To go beyond these drawbacks and improve the method of Ciesla et al. (2018), we consider in the present pilot study a statistical approach, the $\mathrm{ABC}$, combined with a classification algorithm to improve the accuracy and efficiency of their method.

\subsection{Sample}

In this pilot work, we use the wealth of data available on the COSMOS field. The choice of this field is driven by the good spectral coverage of the data and the large statistics of sources.

We drew a sample from the COSMOS catalog of Laigle et al. (2016). A first cut was made to restrict ourselves to galaxies with a stellar mass (Laigle et al. 2016) higher than $10^{8.5} M_{\odot}$. Then we restricted the sample to a relatively narrow redshift range to minimize its effect on the SED and focus our method on the SFH effect on the SED. We therefore selected galaxies with redshifts between 0.5 and 1 , which ensures sufficient statistics in our sample. We used the broadbands of the COSMOS catalog as listed in Table 1. For galaxies with redshifts between 0.5 and 1, Spitzer/IRAC3 probes the 2.9-3.9 $\mu \mathrm{m}$ wavelength range rest 


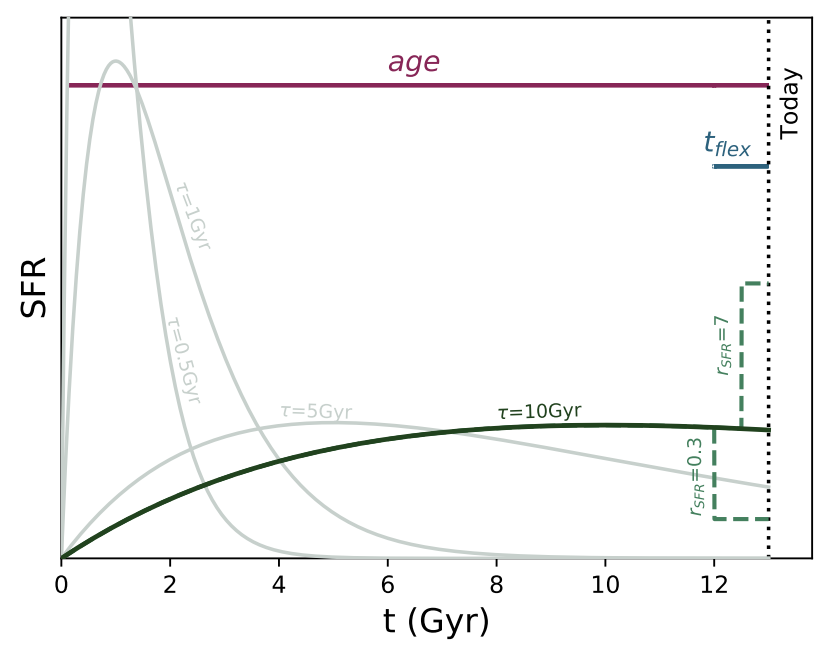

Fig. 1. Examples of delayed- $\tau$ SFHs considered in this work (star formation rate as a function of cosmic time). Different SFHs using $\tau_{\text {main }}=0.5,1,5$, and $10 \mathrm{Gyr}$ are shown to illustrate the effect of this parameter (light green and dark green solid lines). An example of a delayed- $\tau$ SFH with flexibility is shown in solid dark green, with the flexibility as green dashed lines for $\left(\mathrm{age}_{\mathrm{flex}}=1 \mathrm{Gyr}\right.$ and $\left.r_{\mathrm{SFR}}=0.3\right)$ and $\left(\right.$ age $_{\text {flex }}=0.5 \mathrm{Gyr}$ and $\left.r_{\mathrm{SFR}}=7\right)$.

Table 1. COSMOS broadbands.

\begin{tabular}{lcc}
\hline \hline Instrument & Band & $\lambda(\mu \mathrm{m})$ \\
\hline GALEX & FUV & 0.153 \\
GALEX & NUV & 0.229 \\
CFHT & $u^{\prime}$ & 0.355 \\
SUBARU & $B$ & 0.443 \\
SUBARU & $V$ & 0.544 \\
SUBARU & $r$ & 0.622 \\
Suprime Cam & $i^{\prime}$ & 0.767 \\
Suprime Cam & $z^{\prime}$ & 0.902 \\
VISTA & $Y$ & 1.019 \\
VISTA & $J$ & 1.250 \\
VISTA & $H$ & 1.639 \\
VISTA & $K s$ & 2.142 \\
Spitzer & IRAC1 & 3.6 \\
Spitzer & IRAC2 & 4.5 \\
\hline
\end{tabular}

frame and Spitzer/IRAC4 probes the $4-5.3 \mu \mathrm{m}$ range rest frame. These wavelength ranges correspond to the transition between stellar and dust emission. To keep this pilot study simple, we only considered the UV-to-NIR part of the spectrum, which is not affected by dust emission.

One aspect of the ABC method that is still to be developed is handling missing data. In our astrophysical application, we identified several types of missing data. First there is the effect of redshifting, that is, the fact that a galaxy is undetected at wavelengths shorter than the Lyman break at its redshift. Here, the absence of detection provides information on the galaxy coded in its SED. Another type of missing data is linked to the definition of the photometric surveys: the spatial coverage is not exactly the same in every band, and the different sensitivity limits yield undetected galaxies because their fluxes are afaint. To keep the statistical problem simple in this pilot study, we removed galaxies that were not detected in all bands. This strong choice is motivated by the fact that the $\mathrm{ABC}$ method that we use in this pilot study has not been tested and calibrated in the case of missing data such as extragalactic field surveys can produce. The effect of missing data on this method would require much work of statistical research, which is beyond the scope of this paper.

As an additional constraint, we selected galaxies with a signal-to-noise ratio $(\mathrm{S} / \mathrm{N})$ equal or greater than 10 . However, given the importance of the near-UV (NUV) band (Ciesla et al. 2016, 2018) and the faintness of the fluxes compared to the other bands, we relaxed our criteria to an $\mathrm{S} / \mathrm{N}$ of 5 for this band. The first motivation for this cut was again to keep our pilot study simple, but we show in Appendix A that this $\mathrm{S} / \mathrm{N}$ cut is relevant. In the following, we consider a final sample composed of 12380 galaxies for which the stellar mass distribution as a function of redshift is shown in Fig. 2 (top panel) and the distribution of the rejected sources in the bottom panel of the same figure.

The stellar mass distribution, from Laigle et al. (2016), of the final sample is shown in Fig. 3. As a sanity check, we verified that above $10^{9.5} M_{\odot}$, the stellar mass, star formation rate, and specific star formation rate distributions are similar. Our selection criteria mostly affect low-mass galaxies, which is expected because we made $\mathrm{S} / \mathrm{N}$ cuts.

The wide ranges of redshift, stellar masses, and SED shapes we considered create a normalization aspect that needs to be taken into account. This diversity in galaxy properties translates into a large distribution of fluxes in a given photometric band that spans several orders of magnitude: 8 orders of magnitudes in the FUV band and 6 in the $K s$ band, for instance. This parameter space is very challenging for classification algorithms. To avoid this problem, we computed flux ratios. First we combined each flux with the closest one in terms of wavelength. This set of colors provides information on the shape of the SED, but effects of the SFH are also expected on wider scales in terms of wavelength. As discussed in Ciesla et al. (2018), the discrepancy between the UV and NIR emission assuming a smooth delayed$\tau \mathrm{SFH}$ is the signature that we search for because it indicates a possible change in the recent SFH. To be able to probe these effects, we also normalized each photometric band to the $K s$ flux and added this set of colors to the previous one. Finally, we set the flux ratios FUV/NUV and FUV/Ks to be 0 when $z>0.68$ to account for the missing FUV flux density due to the Lyman break at these redshifts.

\section{Statistical approach}

We present the statistical approach that we used to infer the most suitable SFH from photometric data. This new approach is applied to the sample described in Sect. 2.2 as a pilot study, but it can be applied to other datasets and for testing properties other than the SFH.

\subsection{Statistical modeling}

As explained in the previous section, we wish to distinguish between two SFH models: the first is the smooth delayed- $\tau$ SFH, or SFH model $m=0$, and the second is the same with a flexibility in the last $500 \mathrm{Myr}$, or SFH model $m=1$, as presented in Sect. 2.1. The smooth delayed- $\tau$ SFH is thus a specific case of the flexible SFH that is obtained when there is no burst nor quenching $\left(r_{\mathrm{SFR}}=1\right)$.

Let $x_{\mathrm{obs}}$ denote the broadband data collected about a given galaxy. The statistical problem of deciding which SFH fits the data better can be seen as the Bayesian testing procedure distinguishing between both hypotheses,

$H_{0}: r_{\mathrm{SFR}}=1$ versus $H_{1}: r_{\mathrm{SFR}} \neq 1$. 

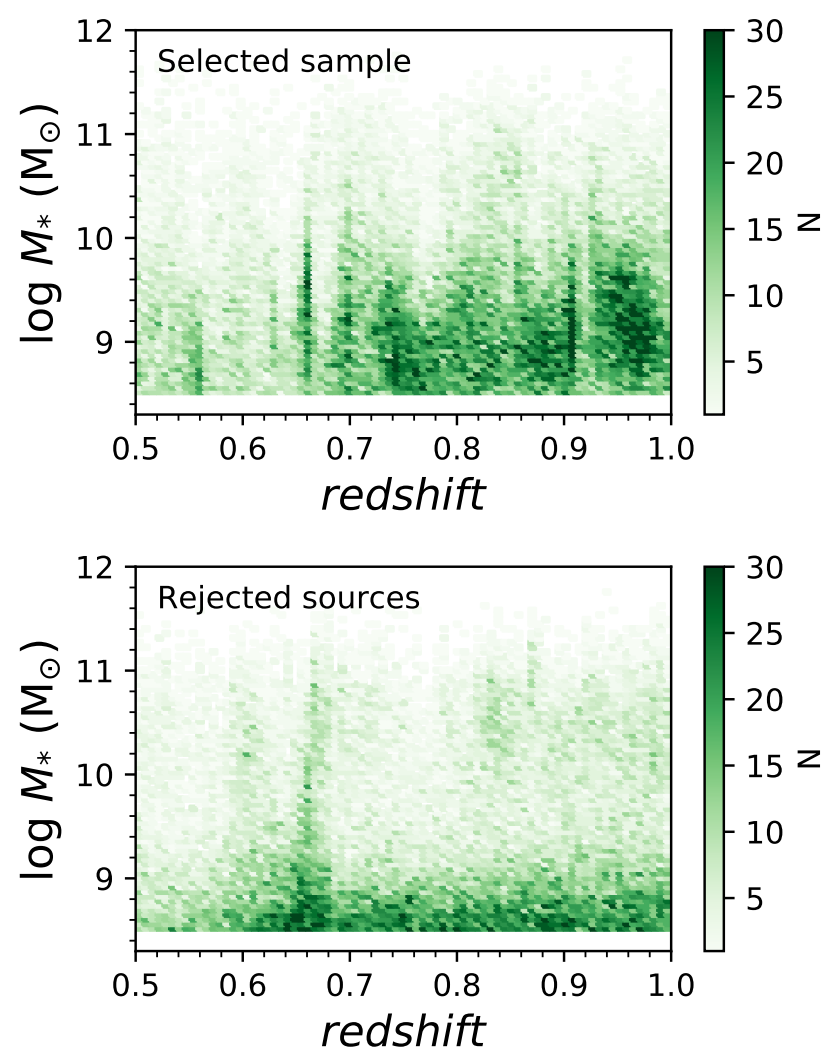

Fig. 2. Stellar mass from Laigle et al. (2016) as a function of redshift for the final sample (top panel) and for the rejected galaxies following our criteria (bottom panel).

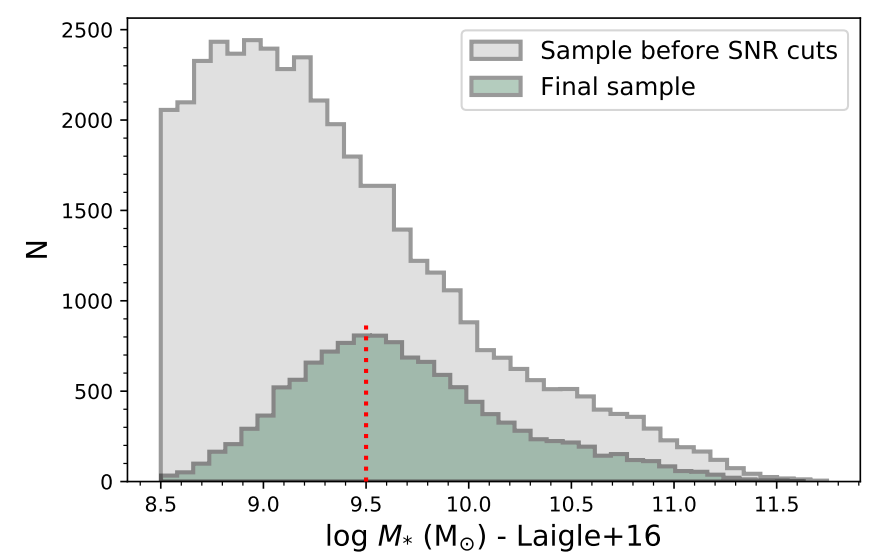

Fig. 3. Distribution of stellar mass for the sample before the $S / N$ cut (gray) and the final sample (green). The red dotted line indicates the limit above which our final sample is considered complete. The stellar masses indicated here are from Laigle et al. (2016).

The procedure decides in favor of a possible change in the recent history when $r_{\mathrm{SFR}}$ is significantly different from 1 based on the data $x_{\text {obs }}$. Conducting a Bayesian testing procedure based on the data $x_{\text {obs }}$ of a given galaxy is exactly the same as the Bayesian model choice that distinguishes between two nested statistical models (Robert 2007).

The first statistical model $(m=0)$, that is, the delayed- $\tau$ SFH, is composed as follow: let $\theta_{0}$ denote the vector of all parameters necessary to compute the mock $\operatorname{SED}$, denoted $\operatorname{SED}\left(\theta_{0}\right)$. In particular, $\theta_{0}$ includes the parameters of the SFH. We denote $p\left(\theta_{0} \mid m=0\right)$ the prior distribution over the parameter space for this statistical model. Likewise for the second SFH model: let $\theta_{1}=\left(\theta_{0}, r_{\mathrm{SFR}}, t_{\mathrm{flex}}\right)$ be the vector of all parameters for the delayed $-\tau+$ flex SFH. This vector includes the same parameters as for the previous SFH, plus two added parameters $r_{\mathrm{SFR}}$ and $t_{\text {flex }}$. Let $p\left(\theta_{1} \mid m=1\right)$ be the prior distribution over the parameter space for the second model. We furthermore add a prior probability on the SFH index, $p(m=1)$ and $p(m=0)$, which are both 0.5 when we wish to remain noninformative.

Finally, we assumed Gaussian noise. Thus, the likelihood $p\left(x_{\mathrm{obs}} \mid \theta_{m}, m\right)$ of $\theta_{m}$ given $x_{\mathrm{obs}}$ under the statistical model $m$ is a multivariate Gaussian distribution, centered on $\operatorname{SED}\left(\theta_{m}\right)$ with a diagonal covariance matrix. The standard deviations were set to $0.1 \times \operatorname{SED}\left(\theta_{m}\right)$ because of the assumed $\mathrm{S} / \mathrm{N}$ value in the observations. In particular, this means that up to constant, the log likelihood is the negative $\chi^{2}$-distance between the observed SED and the mock $\operatorname{SED}\left(\theta_{m}\right)$,

$p\left(x_{\mathrm{obs}} \mid \theta_{m}, m\right) \propto \exp \left(-\frac{1}{2} \chi^{2}\left(x_{\mathrm{obs}}, \operatorname{SED}\left(\theta_{m}\right)\right)\right), \quad$ where
$\chi^{2}\left(x_{\mathrm{obs}}, \operatorname{SED}\left(\theta_{m}\right)\right)=\sum_{j=1}^{J} \frac{\left(x_{\mathrm{obs}}\left(\lambda_{j}\right)-\operatorname{SED}\left(\theta_{m}, \lambda_{j}\right)\right)^{2}}{\left(0.1 \operatorname{SED}\left(\theta_{m}, \lambda_{j}\right)\right)^{2}}$.

\subsection{Bayesian model choice}

The Bayesian model choice (Robert 2007) relies on the evaluation of the posterior probabilities $p\left(m \mid x_{\mathrm{obs}}\right)$ which, using the Bayes formula, is given by

$p\left(m \mid x_{\mathrm{obs}}\right)=\frac{p(m) p\left(x_{\mathrm{obs}} \mid m\right)}{\sum_{m^{\prime}} p\left(m^{\prime}\right) p\left(x_{\mathrm{obs}} \mid m^{\prime}\right)}$,

where

$p\left(x_{\mathrm{obs}} \mid m\right)=\int p\left(x_{\mathrm{obs}} \mid \theta_{m}, m\right) p\left(\theta_{m} \mid m\right) \mathrm{d} \theta_{m}$

is the likelihood integrated over the prior distribution of the $m$ th statistical model. Seen as a function of $x_{\mathrm{obs}}, p\left(x_{\mathrm{obs}} \mid m\right)$ is called the evidence or the integrated likelihood of the $m$ th model.

The Bayesian model choice procedure innately embodies Occam's razor. This principle consists of choosing the simplest model as long as it is sufficient to explain the observation Appendix B. In this study, the two parametric SFHs are nested: when the parameter $r_{\mathrm{SFR}}$ of an SFH $m=1$ (flex + delayed- $\tau$ ) is set to 1, we have an SFH that is also in the model $m=0$ (delayed$\tau)$. Because of Occam's razor, if we choose the SFH with highest posterior probability when analyzing an observed SED $x_{\mathrm{obs}}$ that can be explained by both SFHs, we choose the simplest model $m=0$.

To analyze the dataset $x_{\mathrm{obs}}$, the posterior probabilities remain to be computed. In our situation, the evidence of the statistical model $m$ is intractable. This means that it cannot be easily evaluated numerically. The function that computes $\operatorname{SED}\left(\theta_{m}\right)$ given $m$ and $\theta_{m}$ is fundamentally a black-box numerical function.

There are two methods to solve this problem. First, we can use a Laplace approximation of the integrated likelihood. The resulting procedure chooses the SFH with the smallest BIC. Denoting $\hat{\theta}_{m}$ the maximum likelihood estimate under the SFH $m, \chi^{2}$ the non-reduced $\chi^{2}$-distance of the fit, $k_{m}$ the degree of freedom of model $m$, and $n$ the number of observed photometric bands, the BIC of SFH $m$ is given by

$$
\begin{aligned}
\mathrm{BIC}_{m} & =-2 \max _{\theta_{m}} \ln p\left(x_{\mathrm{obs}} \mid \theta_{m}, m\right)+k_{m} \times \ln (n), \\
& =\chi^{2}\left(\operatorname{SED}\left(\hat{\theta}_{m}\right), x_{\mathrm{obs}}\right)+k_{m} \times \ln (n) .
\end{aligned}
$$


Choosing the model with the smallest BIC is therefore an approximate method to find the model with the highest posterior probability. The results of Ciesla et al. (2018) based on the BIC are justified on this ground. The Laplace approximation assumes, however, that the number of observed photometric bands $n$ is large enough. Moreover, determining the degree of freedom $k_{m}$ of a statistical model can be a complex question. For all these reasons, we expect to improve the method of Ciesla et al. (2018) based on the BIC in the present paper.

Clever Monte Carlo algorithms for computing the evidence, Eq. (6), of each statistical model provide a much sharper approximation of the posterior probabilities of each SFH. We decided to rely on ABC (see, e.g., Marin et al. 2012; Sisson et al. 2018) to compute $p\left(m \mid x_{\mathrm{obs}}\right)$. We could have considered other methods (Vehtari \& Ojanen 2012) such as bridge sampling, reversible jump Markov chain Monte Carlo (MCMC), or nested sampling. these methods require separate runs of the algorithm to analyze each galaxy, however, and probably more than a few minutes per galaxy. We expect to design a faster method here with ABC.

Finally, to interpret the results, we relied on the Bayes factor of the delayed- $\tau+$ flex SFH $(m=1)$ against the delayed- $\tau$ SFH $(m=0)$ given by

$\mathrm{BF}_{1 / 0}\left(x_{\mathrm{obs}}\right)=\frac{p\left(x_{\mathrm{obs}} \mid 1\right)}{p\left(x_{\mathrm{obs}} \mid 0\right)}=\frac{p\left(1 \mid x_{\mathrm{obs}}\right)}{p\left(0 \mid x_{\mathrm{obs}}\right)}=\frac{p\left(1 \mid x_{\mathrm{obs}}\right)}{1-p\left(1 \mid x_{\mathrm{obs}}\right)}$.

The computed value of the Bayes factor was compared to standard thresholds established by Jeffreys (see, e.g., Robert 2007) in order to evaluate the strength of the evidence in favor of delayed$\tau+$ flex SFH if $\mathrm{BF}_{1 / 0}\left(x_{\mathrm{obs}}\right) \geq 1$. Depending on the value of the Bayes factor, Bayesian statisticians are used to say that the evidence in favor of model $m=1$ is either barely worth mentioning (from 1 to $\sqrt{10}$ ), substantial (from $\sqrt{10}$ to 10 ), strong (from 10 to $10^{3 / 2}$ ), very strong (from $10^{3 / 2}$ to 100 ), or decisive (higher than 100).

\section{3. $A B C$ method}

To avoid the difficult computation of the evidence, Eq. (6), of model $m$ and obtain a direct approximation of $p\left(m \mid x_{\mathrm{obs}}\right)$, we resorted to the family of methods called $\mathrm{ABC}$ model choice (Marin et al. 2018).

The main idea behind the ABC framework is that we can avoid evaluating the likelihood and directly estimate a posterior probability by relying on $N$ random simulations $\left(m^{i}, \theta_{m}^{i}, x^{i}\right)$, $i=1, \ldots, N$ from the joint distribution $p(m) p\left(\theta_{m} \mid m\right) p\left(x \mid \theta_{m}, m\right)$. Here simulated $\left(m^{i}, \theta_{m}^{i}, x^{i}\right)$ are obtained as follows: first, we drew an SFH $m^{i}$ at random, with the prior probability $p\left(m^{i}\right)$; then we drew $\theta_{m}^{i}$ according to the prior $p\left(\theta_{m}^{i} \mid m^{i}\right)$; finally, we computed the mock $\operatorname{SED}\left(\theta_{m}^{i}\right)$ with CIGALE and added a Gaussian noise to the mock SED to obtain $x^{i}$. This last step is equivalent to sampling from $p\left(x^{i} \mid \theta_{m}^{i}, m^{i}\right)$ given in Eq. (4). Basically, the posterior distribution $p\left(m \mid x_{\mathrm{obs}}\right)$ can be approximated by the frequency of the SFH $m$ among the simulations that are close enough to $x_{\mathrm{obs}}$.

To measure how close $x$ is from $x_{\text {obs }}$, we introduced the distance between vectors of summary statistics $d\left(S(x), S\left(x_{\mathrm{obs}}\right)\right)$, and we set a threshold $\varepsilon$ : simulations $\left(m, \theta_{m}, x\right)$ that satisfy $d\left(S(x), S\left(x_{\text {obs }}\right)\right) \leq \varepsilon$ are considered "close enough" to $x_{\text {obs }}$. The summary statistics $S(x)$ are primarily introduced as a way to handle feature extraction, whether it is for dimensionality reduction or for data normalization. In this study, the components of the vector $S(x)$ are flux ratios from the SED $x$, chosen for normalization purposes. Mathematically speaking, $p\left(m=1 \mid x_{\mathrm{obs}}\right)$ is thus approximated by
Table 2. Basic ABC model choice algorithm that aims at computing the posterior probabilities of statistical models in competition to explain the data.

$$
\begin{aligned}
& \text { Input: } \\
& -x_{\mathrm{obs}}, \text { the observed SED we want to analyse } \\
& -p(m), \text { prior probability of the } m \text { th statistical model } \\
& -p\left(\theta_{m} \mid m\right), \text { prior distribution of parameter } \theta_{m} \text { of the } m \text { th } \\
& \text { statistical model } \\
& -p\left(x \mid \theta_{m}, m\right), \text { probability density of a SED } x \text { given the } \\
& \\
& \quad m \text { th statistical model, and the parameter } \theta_{m} \text {, see } \\
& \quad \text { Eq. (4) } \\
& -N, \text { number of simulations from the prior } \\
& - \\
& \quad S(x), \text { a function that computes the summary statistics } \\
& \quad \text { of a SED } x
\end{aligned}
$$

\section{Output:}

An approximation $\hat{p}\left(m \mid x_{\mathrm{obs}}\right)$ of the posterior probability of the $m$ th statistical model given the observed data for all $m$.

\section{For $i=1$ to $N$}

2 Generate $m^{i}$ from the prior $p(m)$

3 Generate $\theta_{m}^{i}$ from the prior $p\left(\theta_{m} \mid m\right)$

$4 \quad$ Generate $x^{i}$ from the model $p\left(x \mid \theta_{m}, m\right)$

5 Compute $S\left(x^{i}\right)$ and store $\left(m^{i}, \theta_{m}^{i}, S\left(x^{i}\right)\right)$

6 End For

7 Compute $\hat{p}\left(m \mid x_{\text {obs }}\right)$ with Eq. (8) for all $m$

$\hat{p}\left(m \mid x_{\mathrm{obs}}\right)=\frac{\sum_{i=1}^{N} \mathbf{1}\left\{m^{i}=m\right\} \mathbf{1}\left\{\mathrm{d}\left(S\left(x^{i}\right), S\left(x_{\mathrm{obs}}\right)\right) \leq \varepsilon\right\}}{\sum_{i=1}^{N} \mathbf{1}\left\{\mathrm{d}\left(S\left(x^{i}\right), S\left(x_{\mathrm{obs}}\right)\right) \leq \varepsilon\right\}}$.

The resulting algorithm, called basic ABC model choice, is given in Table 2. Finally, if $k$ is the number of simulations close enough to $x_{\text {obs }}$, the last step of Table 2 can be seen as a $k$ nearestneighbor $(k-n n)$ method that predicts $m$ based on the features (or covariates) $S(x)$.

The $k$-nn can be replaced by other machine-learning algorithms to obtain sharper results. The $k$-nn is known to perform poorly when the dimension of $S(x)$ is larger than 4 . For instance, Pudlo et al. (2016) decided to rely on the method called random forest (Breiman 2001). The machine-learning-based ABC algorithm is given in Table 3. All machine-learning models given below are classification methods. In our context, they separate the simulated datasets $x$ depending on the SFH ( $m=0$ or 1$)$ that was used to generate them. The machine-learning model is fit on the catalog of simulations $\left(m^{i}, \theta_{m}^{i}, x^{i}\right)$, that is to say, it learns how to predict $m$ based on the value of $x$. To this purpose, we fit a function $\hat{p}(m=1 \mid x)$ and performed the classification task on a new dataset $x^{\prime}$ by comparing the fitted $\hat{p}\left(m=1 \mid x^{\prime}\right)$ to $1 / 2$ : if $\hat{p}\left(m=1 \mid x^{\prime}\right)>1 / 2$, the dataset $x^{\prime}$ is classified as generated by SFH $m=1$; otherwise, it is classified as generated by SFH $m=0$. The function $\hat{p}\left(m=1 \mid x^{\prime}\right)$ depends on some internal parameters that are not explicitly shown in the notation. For example, this function can be computed with the help of a neural network. A neuron here is a mathematical function that receives inputs and produces an output based on a weighted combination of the inputs; each neuron processes the received data and transmits its output downstream in the network. Generally, the internal parameters $(\phi, \psi)$ are of two types: the coordinates of $\phi$ are optimized on data with a specific algorithm, and the coordinates of $\psi$ are called tuning parameters (or hyperparameters). For instance, with neural networks, $\psi$ represents the architecture 
of the network and the amount of dropout; $\phi$ represents the collection of the weights in the network.

The gold standard machine-learning practice is to split the catalog of data into three parts: the training catalog and the validation catalog, which are both used to fit the machine-learning models, and the test catalog, which is used to compare the algorithms fairly and obtain a measure of the error committed by the models. Each fit requires two catalogs (training and validation) because modern machine-learning models are fit to the data with a two-step procedure. We detail the procedure for a simple dense neural network and refer to Appendix $\mathrm{C}$ for the general case. The hyperparameters we consider are the number of hidden layers, the number of nodes in each layer, and the amount of dropout. We fixed a range of possible values for each hyperparameter (see Table 4). We selected a possible combination of hyperparameters $\psi$, and trained the obtained neural network on the training catalog. After the weights $\phi$ were optimized on the training catalog, we evaluated the given neural network on the validation catalog and associated the obtained classification error with the combination of hyperparameters that we used. We followed the same training and evaluating procedure for several hyperparameter combinations $\psi$ and selected the one that obtained the lowest classification error. At the end of the process, we evaluated the classification error on the test catalog using the selected combination of hyperparameters $\hat{\psi}$.

The test catalog was left out during the training and the tuning of the machine-learning methods on purpose. The comparison of the accuracy of the approximation that was returned by each machine-learning method on the test catalog ensured a fair comparison between the methods on data unseen during the fit of $\hat{p}_{\hat{\psi}}(m \mid x)$.

In this pilot study, we tried different machine-learning methods and compared their accuracy:

- logistic regression and linear discriminant analysis (Friedman et al. 2001), which are almost equivalent linear models, and serve only as baseline methods,

- neural networks with one or three hidden layers, the core of deep-learning methods that have proved to return sharp results on various signal datasets (images, sounds)

- classification tree boosting (with XGBoost, see Chen \& Guestrin 2016), which is considered a state-of-the-art method in many applied situations, and is often the most accurate algorithm when it is correctly calibrated on a large catalog.

We did not try random forest because it cannot be run on a simulation catalog as large as the one we rely on in this pilot study $\left(N=4 \times 10^{6}\right)$. The motivation of the method we propose is to bypass the heavy computational burden of MCMC-based algorithms to perform a statistical model choice. In this study, random forest was not able to fulfill this aim, unlike the classification methods given above.

\subsection{Building synthetic photometric data}

To compute or fit galaxy SEDs with CIGALE, a list of prior values for each model's parameters is required. The comprehensive module selection in CIGALE allows specifying the SFH entirely, and how the mock SED is computed. The list of prior values for each module's parameters specifies the prior distribution $p\left(\theta_{m} \mid m\right)$. CIGALE uses this list of values or ranges to sample from the prior distribution by picking values on $\theta_{m}$ on a regular grid. This has the inconvenient of being very sensitive to the number of parameters (if $d$ is the number of parameters, and if we assume ten different values for each parameter, the size of the
Table 3. Machine-learning-based ABC model choice algorithm that computes the posterior probability of two statistical models in competition to explain the data.

Input and output: same as Table 2

1 Generate $N$ simulations $\left(m^{i}, \theta_{m}^{i}, x^{i}\right)$ from the joint distribution $p(m) p\left(\theta_{m} \mid m\right) p\left(x \mid \theta_{m}, m\right)$

2 Summarize all simulated datasets (photometric SED) $x^{i}$ with $S\left(x^{i}\right)$ and store all simulated $\left(m^{i}, \theta_{m}^{i}, S\left(x^{i}\right)\right)$ into a large cata$\log$

3 Split the catalog into three parts: training, validation, and test catalogs

4 Fit each machine-learning method on the training and validation catalogs to approximate $p(m=1 \mid S(x))$ with $\hat{p}_{\hat{\psi}}(m=$ $1 \mid x)$

5 Choose the best machine-learning method by comparing their classification errors on the test catalog

6 Return the approximation $\hat{p}\left(m=1 \mid x_{\mathrm{obs}}\right)$ computed with the best method

grid is $10^{d}$ ); producing simulations that are generated with some parameters that are equal. In this study, we instead advocate in favor of drawing values of all parameters at random from the prior distribution, which is uniform over the specified ranges or list of values. The ranges for each model parameter (see Table 4) were chosen to be consistent with those used by Ciesla et al. (2018). In particular, the catalog of simulations drawn at line 1 in Table 3 follows this rule. Each SFH (the simple delayed- $\tau$ or the delayed $-\tau+$ flex) was then convolved with the stellar population models of Bruzual \& Charlot (2003). The attenuation law described in Charlot \& Fall (2000) was then applied to the SED. Finally, CIGALE convolved each mock SED into a COSMOSlike set of filters described in Table 1.

\section{Application to synthetic photometric data}

We first applied our method on simulated photometric data to evaluate its accuracy. The main interest of such synthetic data is that we control all parameters (flux densities, colors, and physical parameters). The whole catalog of simulations was composed of $4 \times 10^{6}$ simulated datasets. We split this catalog at random into three parts, as explained in Sect. 3.3, and added an additional catalog for comparison with CIGALE:

- $3.6 \times 10^{6}$ sources $(90 \%)$ to compose the training catalog,

- 200000 sources $(5 \%)$ to compose the validation catalog,

- 200000 sources $(5 \%)$ to compose the test catalog,

- 30000 additional sources to compose the additional catalog for comparison with CIGALE.

The size of the additional catalog is much smaller to limit the amount of computation time required by CIGALE to run its own algorithm of SED fitting.

\subsection{Calibration and evaluation of the machine-learning methods on the simulated catalogs}

In this section we present the calibration of the machine-learning techniques and their error rates on the test catalog. We then interpret the results given by our method.

As described in Sect. 3.3, we trained and calibrated the machine-learning methods on the training and validation cata$\log$. The results are given in Table 4 . Neither logistic regression nor linear discriminant analysis have tuning parameters that need 
Table 4. Calibration and test of machine-learning methods.

\begin{tabular}{|c|c|c|c|c|}
\hline Method & Tuning parameter & Explored range & Best value & Error rate $(\%)$ \\
\hline Logistic regression & $\emptyset$ & & & 30.27 \\
\hline Linear discriminant analysis & $\emptyset$ & & & 30.43 \\
\hline$k$ nearest-neighbors & $k$ & {$[3600,180000]$} & 5000 & 23.79 \\
\hline One-layer neural network & $\begin{array}{c}\text { Dropout } \\
\text { Nodes in each layer }\end{array}$ & $\begin{array}{l}{[0.1,0.5]} \\
{[16,256]}\end{array}$ & $\begin{array}{r}0.2 \\
128\end{array}$ & $\begin{array}{r}22.51 \\
-\end{array}$ \\
\hline Three-layer neural network & $\begin{array}{c}\text { Dropout } \\
\text { Nodes in each layer }\end{array}$ & $\begin{array}{l}{[0.1,0.5]} \\
{[16,256]}\end{array}$ & $\begin{array}{r}0.2 \\
128\end{array}$ & $\begin{array}{r}21.06 \\
-\end{array}$ \\
\hline Tree boosting (XGBoost) & $\begin{array}{c}\text { Number of trees (nround) } \\
\text { Depth of each tree (max_depth) } \\
\text { Learning rate (eta) }\end{array}$ & $\begin{array}{c}{[100,1000]} \\
{[4,15]} \\
{[0.01,0.2]}\end{array}$ & $\begin{array}{r}400 \\
12 \\
0.1\end{array}$ & $\begin{array}{r}20.98 \\
- \\
-\end{array}$ \\
\hline
\end{tabular}

Notes. The best value of each tuning parameter was found by comparing error rates on the validation catalog. The error rate given in the last column is computed on the test catalog.

Table 5. Prior range of the parameters used to generate the simulation table of SEDs with redshift between 0.5 and 1 .

\begin{tabular}{|c|c|}
\hline Parameter & Value \\
\hline \multicolumn{2}{|c|}{ Delayed- $\tau$ SFH } \\
\hline age (Gyr) & {$[0.5 ; 9]$} \\
\hline$\tau_{\text {main }}(\mathrm{Gyr})$ & {$[0.1 ; 10]$} \\
\hline \multicolumn{2}{|c|}{ Flexible delayed $\tau$ SFH } \\
\hline age (Gyr) & {$[0.5 ; 9]$} \\
\hline$\tau_{\text {main }}(\mathrm{Gyr})$ & {$[0.1 ; 10]$} \\
\hline $\operatorname{age}_{\text {flex }}(\mathrm{Myr})$ & $10,100,450$ \\
\hline $\log r_{\mathrm{SFR}}$ & {$[-6 ; 6]$} \\
\hline \multicolumn{2}{|c|}{ Dust attenuation } \\
\hline$A_{V}$ & {$[0.1 ; 4]$} \\
\hline
\end{tabular}

to be calibrated on the validation catalog. The error rate of these techniques is about $30 \%$ on the test catalog. The modern machine-learning methods $(k-\mathrm{nn}$, neural networks, and tree boosting) were calibrated on the validation catalog, however. The best value of the explored range for $\psi$ was found by comparing error rates on the validation catalog and is given in Table 4. The error rates of these methods on the test catalog vary between $24 \%$ and $20 \%$. The significant gain in using nonlinear methods is therefore clear. However, we see no obvious use in training a more complex algorithm (such as a deeper neural network) for this problem, although it might become useful when the number of photometric bands and the redshift range are increased. Finally, we favor XGBoost for our study. While neural networks might be tuned more precisely to match or exceed its performances, we find XGBoost easier to tune and interpret.

Machine-learning techniques that fit $\hat{p}_{\hat{\psi}}(m \mid x)$ are often affected by some bias and may require some correction (Niculescu-Mizil \& Caruana 2012). These classification algorithms compare the estimated probabilities of $m$ given $x$ and return the most likely $m$ given $x$. The output $m$ can be correct even if the probabilities are biased toward 0 for low probabilities or toward 1 for high probabilities. A standard reliability check shows no such problem for our XGBoost classifier. To this aim, the test catalog was divided into ten bins: the first bin is composed of simulations with a predicted probability $\hat{p}\left(m=1 \mid x_{\mathrm{obs}}\right)$ between 0 and 0.1 , the second with $\hat{p}\left(m=1 \mid x_{\text {obs }}\right)$ between 0.1 and 0.2 etc. The reliability check procedure ensures that the frequency of the SFH $m=1$ among the $k$ th bin falls within the range $[(k-1) / 10 ; k / 10]$ because the $\hat{p}\left(m=1 \mid x_{\text {obs }}\right)$ predicted by XGBoost are between $(k-1) / 10$ and $k / 10$.

We studied the ability of our method to distinguish the SFH of the simulated test-catalog sources. The top panel of Fig. 4 shows the distribution of $\hat{p}\left(m=1 \mid x_{\mathrm{obs}}\right)$ when $x$ varies in the test catalog. Naively, a perfect result would have half of the sample with $p=1$ and the other half with $p=0$. When $m=0$, the SFH $m=1$ is also suitable because the models are nested. In this case, Occam's razor favors the model $m=0$, and $\hat{p}\left(m=1 \mid x_{\text {obs }}\right)$ must be lower than 0.5 , see Sect. 3.2. In contrast, for the SEDs that are explained by the SFH model $m=1$ alone, $\hat{p}\left(m=1 \mid x_{\mathrm{obs}}\right)$ is close to 1 .

The distribution (Fig. 4, bottom left panel) has two peaks, one centered around $p=0.2$ and one between 0.97 and 1 . This peak at 0.2 , and not 0 , is expected when one of the models proposed to the choice is included in the second model. In the distribution of the $\hat{p}\left(m=1 \mid x_{\mathrm{obs}}\right), 20 \%$ of the sources have a value higher than 0.97 and $52 \%$ lower than 0.4 . In the right panels of Fig. 4 we show the distribution of $r_{\mathrm{SFR}}$ for the galaxies $x$ with $\hat{p}\left(m=1 \mid x_{\text {obs }}\right)>0.97$. With a perfect method, galaxies with $r_{\mathrm{SFR}} \neq 1$ should have $\hat{p}\left(m=1 \mid x_{\mathrm{obs}}\right)=1$. Here we see indeed a deficit of galaxies around $p=1$, but the range of affected $r_{\mathrm{SFR}}$ extends from 0.1 to 10 . This shows that the method is not able to identify galaxies with an SFR variability if this variability is only $0.1-10$ times the SFR before the variability began. In other words, the method is sensitive to $\left|\log r_{\mathrm{SFR}}\right|>1$. This is confirmed by the distribution of $r_{\mathrm{SFR}}$ for galaxies with $p<0.40$ (Fig. 4, bottom panel). However, there are sources with a $\left|\log r_{\mathrm{SFR}}\right|>1$ that is associated with low values of $\hat{p}\left(m=1 \mid x_{\mathrm{obs}}\right)$. The complete distribution of $r_{\mathrm{SFR}}$ as a function of $\hat{p}\left(m=1 \mid x_{\mathrm{obs}}\right)$ is shown in Fig. 4.

\subsection{Importance of particular flux ratios}

We determined which part of the dataset $x$ most influences the choice of SFH given by our method. The analysis of $x$ relies entirely on the summary statistics $S(x)$, the flux ratios. We 

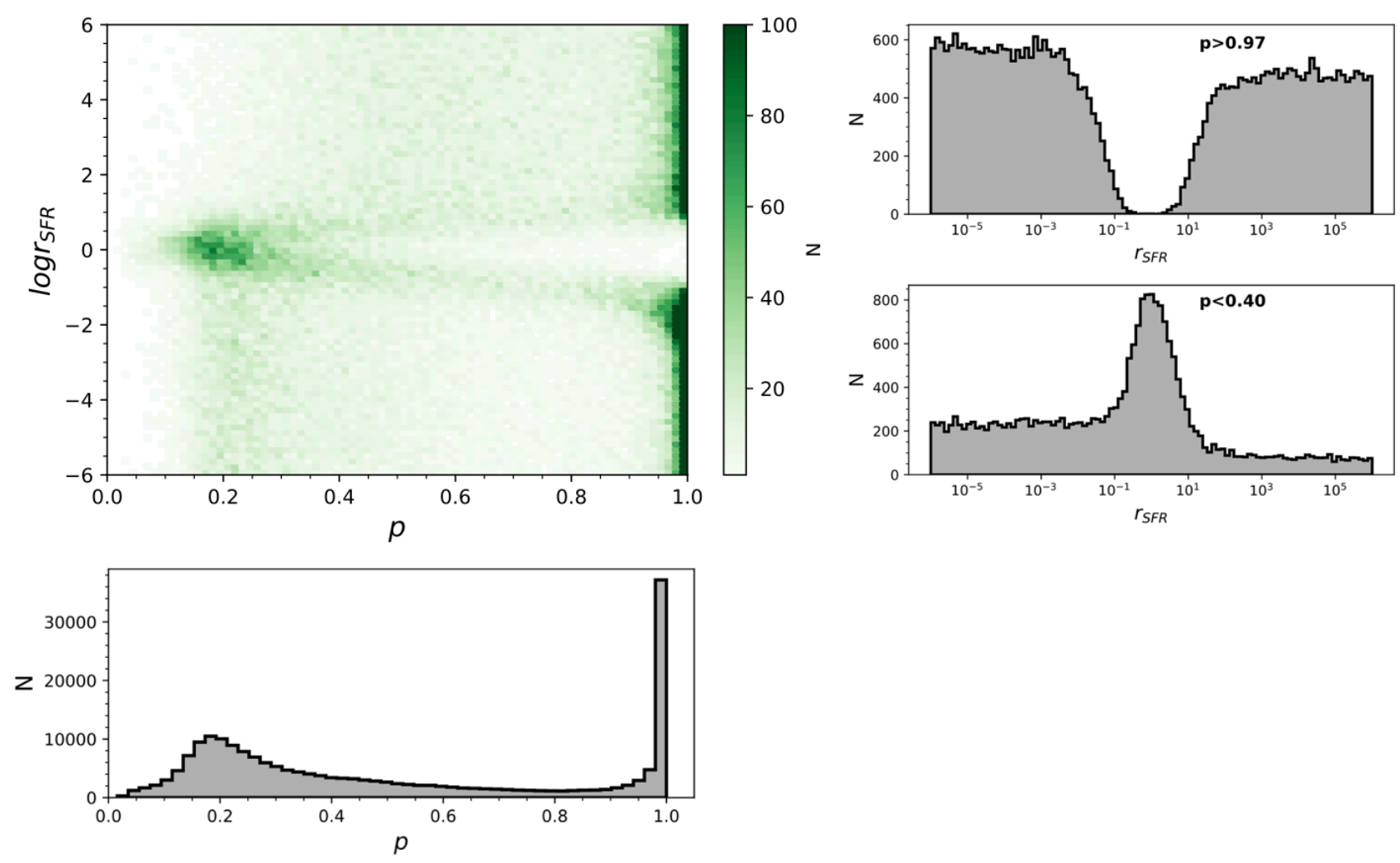

Fig. 4. Study of the statistical power of $\hat{p}\left(m=1 \mid x_{\mathrm{obs}}\right)$ to detect short-term variations with respect to the value of $r_{\mathrm{SFR}}$. Top left panel: joint distribution of $\hat{p}\left(m=1 \mid x_{\mathrm{obs}}\right)$ and $r_{\mathrm{SFR}}$. Bottom left panel: distribution of $\hat{p}\left(m=1 \mid x_{\mathrm{obs}}\right)$ obtained with $x$ coming from the test catalog. Right panels: marginal distributions of $r_{\mathrm{SFR}}$ for mock sources with $\hat{p}\left(m=1 \mid x_{\mathrm{obs}}\right)>0.97$ (top right panel) and for mock sources with $\hat{p}\left(m=1 \mid x_{\mathrm{obs}}\right)<0.4($ bottom right panel).

therefore tried to understand which flux ratios are most discriminant for the model choice. We wished to verify that the method is not based on a bias of our simulations and to assess which part of the data could be removed without losing crucial information.

We used different usual metrics (e.g., Friedman et al. 2001; Chen \& Guestrin 2016) to assess the importance of each flux ratio in the machine-learning estimation of $\hat{p}(m=1 \mid x)$. These metrics are used as indicators of the relevance of each flux ratio for the classification task. As expected, the highest flux ratios for our problem involve the bands at shortest wavelength (FUV at $z<0.68$ and NUV above because FUV is no longer available), normalized by either $K s$ or $u$. This is expected because these bands are known to be sensitive to SFH (e.g., Arnouts et al. 2013). We see no particular pattern in the estimated importance of the other flux ratios. They were all used for the classification, and removing any of them decreases the classification accuracy, except for IRAC $1 / K s$, whose importance is consistently negligible across every considered metric.

We also tested whether the UVJ selection we used to classify galaxies according to their star formation activity (e.g., Wuyts et al. 2007; Williams et al. 2009) is able to probe the type of rapid and recent $\mathrm{SFH}$ variations we investigate here. We trained an XGBoost classification model using only $u / V$ and $V / J$ in order to evaluate the benefits of using all available flux ratios. This resulted in a severe increase in classification error, which increased from $21.0 \%$ using every flux ratios to $35.8 \%$.

\subsection{Comparison with SED fitting methods based on the BIC}

In this section we compare the results obtained with the $\mathrm{ABC}$ method to those obtained with a standard SED modeling. The goal of this test is to understand and quantify the improvement that the $\mathrm{ABC}$ method brings in terms of result accuracy. We used the simulated catalog of 30000 sources, described at the beginning of this section, for which we controlled all parameters.

The ABC method was also used on this additional catalog. This test is very similar to the training procedure described in Sect. 4.1. With this additional catalog, the ABC method has an error rate of $21.2 \%$ compared to $21.0 \%$ with the previous test sample.

CIGALE was run on the test catalog as well. The set of modules was the same as the set we used to create the mock SEDs, but the parameters we used to fit the test catalog did not include the input parameters, which were chosen randomly. This test was intentionally thought to be simple and represent an ideal case scenario. The error rate that was obtained with CIGALE therefore represents the best achievable result.

To decide whether a flexible SFH was preferable to a normal delayed- $\tau$ SFH using CIGALE, we adopted the method of Ciesla et al. (2018) described in Sect. 2.1. The quality of fit using each SFH was tested through the use of the BIC.

In detail, the method we used was the following: First, we performed a run with CIGALE using a simple delayed- $\tau$ SFH whose parameters are presented in Table 6. A second run was then performed with the flexible SFH. We compared the results and quality of the fits using one SFH or the other. The two models have different numbers of degrees of freedom. To take this into account, we computed the BIC presented in Sect. 3.2 for each SFH.

We then calculated the difference between $\mathrm{BIC}_{\text {delayed }}$ and $\mathrm{BIC}_{\text {flex }}(\triangle \mathrm{BIC})$ and used the threshold defined by Jeffreys 
Table 6. Input parameters used in the SED fitting procedures with CIGALE.

\begin{tabular}{lc}
\hline \hline Parameter & Value \\
\hline & Delayed $-\tau$ SFH \\
age $(\mathrm{Gyr})$ & {$[0.5 ; 9], 15$ values linearly sampled } \\
$\tau_{\text {main }}(\mathrm{Gyr})$ & {$[0.1 ; 10], 15$ values linearly sampled } \\
\hline & Flexible delayed $-\tau$ SFH \\
age $(\mathrm{Gyr})$ & {$[0.5 ; 9], 15$ values linearly sampled } \\
$\tau_{\text {main }}(\mathrm{Gyr})$ & {$[0.1 ; 10], 15$ values linearly sampled } \\
age & flex $(\mathrm{Myr})$ \\
$\log r_{\mathrm{SFR}}$ & {$[-6 ; 100,450$} \\
\hline & Dust attenuation \\
$A_{V}^{\mathrm{ISM}}$ & {$[0.1 ; 4], 10$ values linearly sampled } \\
\hline
\end{tabular}

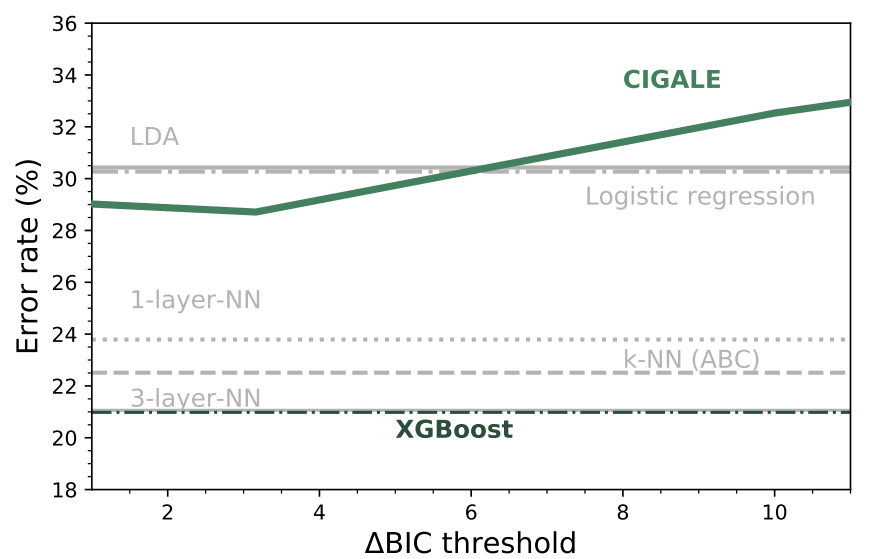

Fig. 5. Error rate obtained with CIGALE as a function of the $\triangle B I C$ chosen threshold. For comparison we show the error rates obtained by the classification methods tested in Sect. 3.

(Sect. 3.2), which is valid either for the BF and the BIC and was also used in Ciesla et al. (2018): a $\triangle \mathrm{BIC}$ larger than 10 is interpreted as a strong difference between the two fits (Kass \& Raftery 1995), with the flexible SFH providing a better fit of the data than the delayed- $\tau$ SFH.

We applied this method to the sample containing $15 \mathrm{k}$ sources modeled with a delayed- $\tau$ SFH and $15 \mathrm{k}$ modeled using a delayed- $\tau$ + flexibility. With these criteria, we find that the error rate of CIGALE, in terms of identifying SEDs built with a delayed $-\tau+$ flex $\mathrm{SFH}$, is $32.5 \%$. This rate depends on the $\triangle \mathrm{BIC}$ threshold chosen and increases with the value of the threshold, as shown in Fig. 5. The best value, 28.7\%, is lower than the error rate obtained from a logistic regression or an LDA (see Table 4) but is significantly higher than the error rate obtained from our procedure using XGBoost $(21.0 \%)$. In this best-case scenario test for CIGALE, a difference of $7.7 \%$ is substantial and implies that the ABC method tested in this study provides better results than a more traditional one using SED fitting. When considering sources with $\triangle \mathrm{BIC}>10$, that is, sources for which the method using CIGALE estimates that there is strong evidence for the flexible SFH, 95.4\% are indeed SEDs simulated with the flexible SFH. Using our procedure with XGBoost and the Bayes factor corresponding threshold of 150 (Kass \& Raftery 1995), we find that $99.7 \%$ of the source SFHs are correctly identified. The ABC method provides a cleaner sample than the CIGALE $\triangle \mathrm{BIC}$-based method.

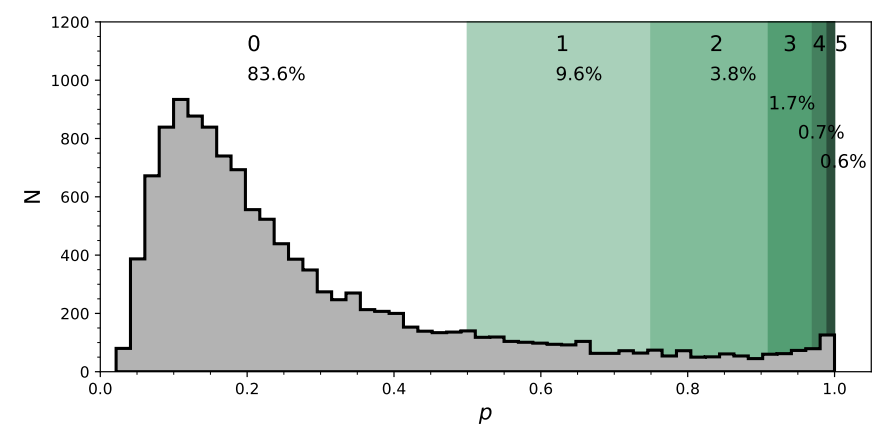

Fig. 6. Distribution of the predictions $\hat{p}\left(m=1 \mid x_{\mathrm{obs}}\right)$ produced by our algorithm on the selected COSMOS data. Sources with a $\hat{p}\left(m=1 \mid x_{\mathrm{obs}}\right)$ close to 1 tend to prefer the delayed- $\tau+$ flex SFH, while sources with lower $\hat{p}\left(m=1 \mid x_{\mathrm{obs}}\right)$ favor a simple delayed- $\tau$ SFH. The green regions numbered from 1 to 5 indicate the Jeffreys scale of the Bayes factor, 1: barely worth mentioning, 2: substantial, 3: strong, 4: very strong, and 5: decisive (detailed at the end of Sect. 3.2). The percentage of sources in each grade is provided in the figure and in Table 7.

Table 7. Jeffreys scale and statistics of our sample.

\begin{tabular}{llcc}
\hline \hline Grade & Evidence against delayed- $\tau$ SFH & Number & $\%$ \\
\hline 1 & Barely worth mentioning & 1187 & 9.6 \\
2 & Substantial & 466 & 3.8 \\
3 & Strong & 209 & 1.7 \\
4 & Very strong & 90 & 0.7 \\
5 & Decisive & 77 & 0.6 \\
\hline
\end{tabular}

\section{Application on COSMOS data}

We now apply our method to the sample of galaxies drawn from the COSMOS catalog, whose selection is described in Sect. 2.2. As a result, we show the $\hat{p}\left(m=1 \mid x_{\text {obs }}\right)$ distribution obtained for this sample of observed galaxies in Fig. 6. We recall that the 0 value indicates that the delayed- $\tau$ SFH is preferred, whereas $\hat{p}=1$ indicates that the flexible SFH is more adapted to fit the SED of the galaxy. As a guide, we indicate the different grades of the Jeffreys scale and provide the number of sources in each grade in Table 7. The flexible SFH models the observations of $16.4 \%$ of our sample better than the delayed- $\tau$ SFH. However, this also means that for most of the dataset $(83.6 \%)$, there is no strong evidence for a necessity to increase the complexity of the $\mathrm{SFH}$; a delayed- $\tau$ is sufficient to model the SED of these sources.

To investigate the possible differences in terms of physical properties of galaxies according to their Jeffreys grade, we divided the sample of galaxies into two groups. The first group corresponds to galaxies with $\hat{p}\left(m=1 \mid x_{\mathrm{obs}}\right)<0.5$, galaxies for which there is no evidence for the need of a recent burst or quenching in the SFH, a delayed- $\tau$ SFH is sufficient to model the SED of these sources. We selected the galaxies of the second group imposing $\hat{p}\left(m=1 \mid x_{\text {obs }}\right)>0.75$, that is, Jeffreys scale grades of 3,4 , or 5 : from strong to decisive evidence against the normal delayed- $\tau$. In Fig. 7 (top panel) we show the stellar mass distribution of the two subsamples. Although the stellar masses obtained with either the smooth delayed- $\tau$ or the flexible SFH are consistent with each other, for each galaxy we used the most suitable stellar mass: if the galaxy had $\hat{p}\left(m=1 \mid x_{\mathrm{obs}}\right)<0.5$, the stellar mass obtained from the delayed- $\tau$ SFH was used, and if the galaxy had $\hat{p}\left(m=1 \mid x_{\mathrm{obs}}\right)>0.75$, the stellar mass obtained with the flexible SFH was used. The stellar mass distribution of galaxies with a delayed- $\tau$ SFH is similar to the distribution of the 



Fig. 7. Top panel: comparison of the stellar mass distribution obtained with CIGALE for the sample of galaxies with $\hat{p}\left(m=1 \mid x_{\text {obs }}\right)>=0.75$ (green) and galaxies with $\hat{p}\left(m=1 \mid x_{\mathrm{obs}}\right)<0.5$ (gray). Middle panel: comparison of the stellar mass distribution obtained by Laigle et al. (2016) for the sample of galaxies with $\hat{p}\left(m=1 \mid x_{\mathrm{obs}}\right)>=0.75$ (green) and galaxies with $\hat{p}\left(m=1 \mid x_{\mathrm{obs}}\right)<0.5$ (gray). Bottom panel: comparison of the sSFR distribution for the sample of galaxies with $\hat{p}(m=$ $\left.1 \mid x_{\mathrm{obs}}\right)>=0.75$ (green) and galaxies with $\hat{p}\left(m=1 \mid x_{\mathrm{obs}}\right)<0.5$ (gray).

whole sample, as shown in the middle panel of Fig. 7. However, the stellar mass distribution of galaxies needing a flexibility in their recent SFH shows a deficit of galaxies with stellar masses between $10^{9.5}$ and $10^{10.5} M_{\odot}$ compared to the distribution of the fool sample. We note that at masses hiher than $10^{10.5} M_{\odot}$ the distribution are identical, despite a small peak at $10^{11.1} M_{\odot}$. To verify that this results is not due to our SED modeling procedure and the assumptions we adopted, we show in the middle panel of Fig. 7 the same stellar mass distributions, this time using the values published by Laigle et al. (2016). The two stellar mass distributions, with the one of galaxies with $\hat{p}\left(m=1 \mid x_{\text {obs }}\right)>0.75$ peaking at a lower mass, are recovered. This implies that these differences between the distributions are independent of the SED fitting method that is employed to determine the stellar mass of the galaxies. We note that when the algorithm has been trained, only ratios of fluxes were provided to remove the normalization factor out of the method, and the mock SEDs from which the flux ratios were computed were all normalized to $1 M_{\odot}$. The stellar mass is at first order a normalization through, for instance, the $L_{K}-M_{*}$ relation (e.g., Gavazzi et al. 1996). When flux ratios were used, the algorithm had no information linked to the stellar mass of the mock galaxies. Nevertheless, applied to real galaxies, the result of our procedure yields two different stellar mass distributions between galaxies identified as having smooth SFH and galaxies undergoing a more drastic episode (star formation burst or quenching).

In the bottom panel of Fig. 7 we show the distribution in specific star formation rate $\left(\mathrm{sSFR}, \mathrm{sSFR} \equiv \mathrm{SFR} / M_{*}\right.$ ) for the same two samples. The distribution of galaxies with $\hat{p}\left(m=1 \mid x_{\mathrm{obs}}\right)<$ 0.5 is narrow $(\sigma=0.39)$ and has one peak at log SSFR = $-0.32\left(\mathrm{Gyr}^{-1}\right)$, clearly showing the MS of star-forming galaxies. Galaxies with a high probability to have a recent strong variation in their SFH form a double-peaked distribution with one peak above the MS that is formed by galaxies with $\hat{p}\left(m=1 \mid x_{\mathrm{obs}}\right)>$ 0.75 (log sSFR $=0.66)$, corresponding to galaxies having experienced a recent burst, and a second peak at lower sSFRs than the MS, corresponding to sources having undergone a recent decrease in their star formation activity $(\log \mathrm{SSFR}=-1.38)$. In the sample of galaxies with $\hat{p}\left(m=1 \mid x_{\mathrm{obs}}\right)>0.75,28 \%$ of these sources are in the peak of galaxies experiencing a burst of star formation activity and $72 \%$ seem to undergo a rapid and drastic decrease of their SFR. One possibility to explain this asymmetry could be a bias produced by the algorithm, as shown in Fig. 4, more sources with $\hat{p}\left(m=1 \mid x_{\mathrm{obs}}\right)>0.97$ tend to be associated with low values of $r_{\mathrm{SFR}}$ than with $r_{\mathrm{SFR}}>1$. However, in the case of the additional catalog, this disparity is $47 \%$ and $53 \%$ for high and low $r_{\mathrm{SFR}}$, respectively.

The distribution of the two samples in terms of SSFR indicates that to be able to reach the sSFR of galaxies that are outside the MS, a flexibility in the SFH of galaxies had to be taken into acount when the SED modeling was performed. This is needed to recover the parameter space in SFR and $M_{*}$ as far as possible.

\section{Conclusions}

In this pilot study, we proposed to use a custom statistical method using a machine-learning algorithm, the approximate Bayesian computation, to determine the best-suited SFH to be used to measure the physical properties of a subsample of COSMOS galaxies. These galaxies were selected in mass $\left(\log M_{*}>\right.$ 8.5) and redshift $(0.5<z<1)$. Furthermore, we imposed that the galaxies should be detected in all UV-to-NIR bands with an $\mathrm{S} / \mathrm{N}$ higher than 10 . We verified that these criteria do not bias the sSFR distribution of the sample.

To model these galaxies, we considered a smooth delayed- $\tau$ SFH with or without a rapid and drastic change in the recent $\mathrm{SFH}$, that is, in the last few hundred million years. We built a mock galaxy SED using the SED-fitting code CIGALE. The mock SEDs were integrated into the COSMOS set of broadband filters. To avoid large dynamical ranges of fluxes, which is to be avoided when classification algorithms are used, we computed flux ratios.

Different classification algorithms were tested with XGBoost and provided the best results with a classification error of $20.98 \%$. As output, the algorithm provides the probability that a galaxy is better modeled using a flexibility in the recent SFH. The method is sensitive to variations in SFR that are larger than 1 dex.

We compared the results from the ABC new method with SED-fitting using CIGALE. Following the method proposed by Ciesla et al. (2018), we compared the results of two SED fits, one using the delayed- $\tau$ SFH and the other adding a flexibility in the recent history of the galaxy. The BIC was computed and compared to determine which SFH provided a better fit. The BIC method provides a high error rate, $28 \%$, compared to the 
$21 \%$ obtained with the ABC method. Moreover, because the BIC method requires two SED fits per analysis of a source, it is much slower than the proposed ABC method: we were not able to compare them on the test catalog of 200000 sources, and we had to introduce a smaller simulated catalog of 30000 sources to compute their BIC in a reasonable amount of time.

We used the result of the ABC method to determine the stellar mass and SFRs of the galaxies using the best-suited SFH for each of them. We compared two samples of galaxies: the first was galaxies with $\hat{p}\left(m=1 \mid x_{\text {obs }}\right)<0.5$, which are galaxies for which the smooth delayed- $\tau$ SFH is preferred, the second sample was galaxies with $\hat{p}\left(m=1 \mid x_{\text {obs }}\right)>0.75$, that is, galaxies for which there is strong to decisive evidence against the smooth delayed- $\tau$ SFH. The stellar mass distribution of these two samples is different. The mass distribution of galaxies for which the delayed- $\tau$ SFH is preferred is similar to the distribution of the whole sample. However, the mass distribution of galaxies that required a flexible SFH shows a deficit between $10^{9.5}$ and $10^{10.5} M_{\odot}$. Their distribution is similar to that of the whole sample above $M_{*}=10^{10.5} M_{\odot}$, however. Furthermore, the results of this study also imply that a flexible $\mathrm{SFH}$ is required to cover the largest parameter space in terms of stellar mass and SFR, as seen from the sSFR distributions of galaxies with $\hat{p}\left(m=1 \mid x_{\mathrm{obs}}\right)>0.75$.

Acknowledgements. The authors thank Denis Burgarella and Yannick Roehlly for fruitful discussions and the referee for his valuable comments that helped improve the paper. The research leading to these results was partially financed via the PEPS Astro-Info program of the CNRS. P. Pudlo warmly thanks the Centre International de Rencontres Mathématiques (CIRM) of Aix-Marseille University for its support and the stimulating atmosphere during the Jean Morlet semester "Bayesian modeling and Analysis of Big Data" chaired by Kerrie Mengersen.

\section{References}

Abramson, L. E., Gladders, M. D., Dressler, A., et al. 2016, ApJ, 832, 7 Arnouts, S., Le Floc'h, E., Chevallard, J., et al. 2013, A\&A, 558, A67 Behroozi, P. S., Wechsler, R. H., \& Conroy, C. 2013, ApJ, 770, 57 Boquien, M., Buat, V., \& Perret, V. 2014, A\&A, 571, A72 Boquien, M., Burgarella, D., Roehlly, Y., et al. 2019, A\&A, 622, A103 Boselli, A., Roehlly, Y., Fossati, M., et al. 2016, A\&A, 596, A11 Breiman, L. 2001, Mach. Learn., 45, 5 Bruzual, G., \& Charlot, S. 2003, MNRAS, 344, 1000

Buat, V., Heinis, S., Boquien, M., et al. 2014, A\&A, 561, A39 Calzetti, D., Armus, L., Bohlin, R. C., et al. 2000, ApJ, 533, 682 Carnall, A. C., Leja, J., Johnson, B. D., et al. 2019, ApJ, 873, 44
Charlot, S., \& Fall, S. M. 2000, ApJ, 539, 718

Chen, T., \& Guestrin, C. 2016, Proceedings of the 22nd ACM SIGKDD International Conference on Knowledge Discovery and Data Mining (ACM), 785

Ciesla, L., Charmandaris, V., Georgakakis, A., et al. 2015, A\&A, 576, A10

Ciesla, L., Boselli, A., Elbaz, D., et al. 2016, A\&A, 585, A43

Ciesla, L., Elbaz, D., \& Fensch, J. 2017, A\&A, 608, A41

Ciesla, L., Elbaz, D., Schreiber, C., Daddi, E., \& Wang, T. 2018, A\&A, 615, A61

Dekel, A., \& Burkert, A. 2014, MNRAS, 438, 1870

Elbaz, D., Daddi, E., Le Borgne, D., et al. 2007, A\&A, 468, 33

Friedman, J., Hastie, T., \& Tibshirani, R. 2001, The Elements of Statistical Learning (New York: Springer-Verlag)

Gavazzi, G., Pierini, D., \& Boselli, A. 1996, A\&A, 312, 397

Gladders, M. D., Oemler, A., Dressler, A., et al. 2013, ApJ, 770, 64

Guo, K., Zheng, X. Z., \& Fu, H. 2013, ApJ, 778, 23

Ilbert, O., Arnouts, S., Le Floc'h, E., et al. 2015, A\&A, 579, A2

Kass, R. E., \& Raftery, A. E. 1995, J. Am. Stat. Assoc., 90, 773

Laigle, C., McCracken, H. J., Ilbert, O., et al. 2016, ApJS, 224, 24

Lee, S.-K., Ferguson, H. C., Somerville, R. S., Wiklind, T., \& Giavalisco, M. 2010, ApJ, 725, 1644

Leja, J., Carnall, A. C., Johnson, B. D., Conroy, C., \& Speagle, J. S. 2019, ApJ, 876,3

Magdis, G. E., Daddi, E., Béthermin, M., et al. 2012, ApJ, 760, 6

Maraston, C. 2005, MNRAS, 362, 799

Maraston, C., Pforr, J., Renzini, A., et al. 2010, MNRAS, 407, 830

Marin, J.-M., Pudlo, P., Robert, C. P., \& Ryder, R. J. 2012, Stat. Comput., 22 1167

Marin, J. M., Pudlo, P., Estoup, A., \& Robert, C. 2018, in Handbook of Approximate Bayesian Computation, eds. S. A. Sisson, Y. Fan, \& M. Beaumont (Chapman and Hall/CRC)

Niculescu-Mizil, A., \& Caruana, R. 2012, ArXiv e-prints [arXiv:1207. 1403]

Noeske, K. G., Weiner, B. J., Faber, S. M., et al. 2007, ApJ, 660, L43

Pacifici, C., Kassin, S. A., Weiner, B., Charlot, S., \& Gardner, J. P. 2013, ApJ, 762, L15

Pacifici, C., Oh, S., Oh, K., Lee, J., \& Yi, S. K. 2016, ApJ, 824, 45

Papovich, C., Dickinson, M., \& Ferguson, H. C. 2001, ApJ, 559, 620

Pforr, J., Maraston, C., \& Tonini, C. 2012, MNRAS, 422, 3285

Pudlo, P., Marin, J.-M., Estoup, A., et al. 2016, Bioinformatics, 32, 859

Robert, C. 2007, The Bayesian Choice: From Decision Theoretic Foundations to Computational Implementation (Springer Science \& Business Media)

Salmi, F., Daddi, E., Elbaz, D., et al. 2012, ApJ, 754, L14

Sargent, M. T., Daddi, E., Béthermin, M., et al. 2014, ApJ, 793, 19

Schreiber, C., Pannella, M., Elbaz, D., et al. 2015, A\&A, 575, A74

Scoville, N., Sheth, K., Aussel, H., et al. 2016, ApJ, 820, 83

Simha, V., Weinberg, D. H., Conroy, C., et al. 2014, ArXiv e-prints [arXiv:1404.0402]

Sisson, S. A., Fan, Y., \& Beaumont, M. 2018, Handbook of Approximate Bayesian Computation (Chapman and Hall/CRC)

Tacchella, S., Dekel, A., Carollo, C. M., et al. 2016, MNRAS, 458, 242

Vehtari, A., \& Ojanen, J. 2012, Stat. Surv., 6, 142

Williams, R. J., Quadri, R. F., Franx, M., van Dokkum, P., \& Labbé, I. 2009, ApJ, 691, 1879

Wuyts, S., Labbé, I., Franx, M., et al. 2007, ApJ, 655, 51

Wuyts, S., Förster Schreiber, N. M., van der Wel, A., et al. 2011, ApJ, 742, 96 


\section{Appendix A: Effect of flux $\mathrm{S} / \mathrm{N}$ on the distribution of $p\left(x_{0 b s} \mid m=1\right)$}

In Fig. A.1 we show the distribution of the estimated probability $\hat{p}\left(m=1 \mid x_{\mathrm{obs}}\right)$ for the subsample of COSMOS sources described in Sect. 2.2 before any $\mathrm{S} / \mathrm{N}$ cuts are applied. In this figure, all COSMOS sources with $M_{*}>10^{8.5} M_{\odot}$ and redshift between 0.5 and 1 are used. The 0 value indicates that the delayed- $\tau$ SFH is preferred, whereas $\hat{p}=1$ indicates that the delayed $-\tau+$ flex $\mathrm{SFH}$ is more adapted to fit the SED of the galaxy. To understand what drives the shape of the $\hat{p}\left(m=1 \mid x_{\mathrm{obs}}\right)$ distribution, we show in the same figure the distributions obtained for different $K s \mathrm{~S} / \mathrm{N}$ bins (top panel) and NUV S/N bins (bottom panel). Galaxies with low S/N in either NUV and $K s$ photometric band show flatter $\hat{p}\left(m=1 \mid x_{\mathrm{obs}}\right)$ distributions. This means that these low $\mathrm{S} / \mathrm{N}$ sources yield intermediate values of $\hat{p}\left(m=1 \mid x_{\mathrm{obs}}\right)$, translating into a difficulty of choosing between the delayed- $\tau$ and the delayed $-\tau+$ flex SFHs.
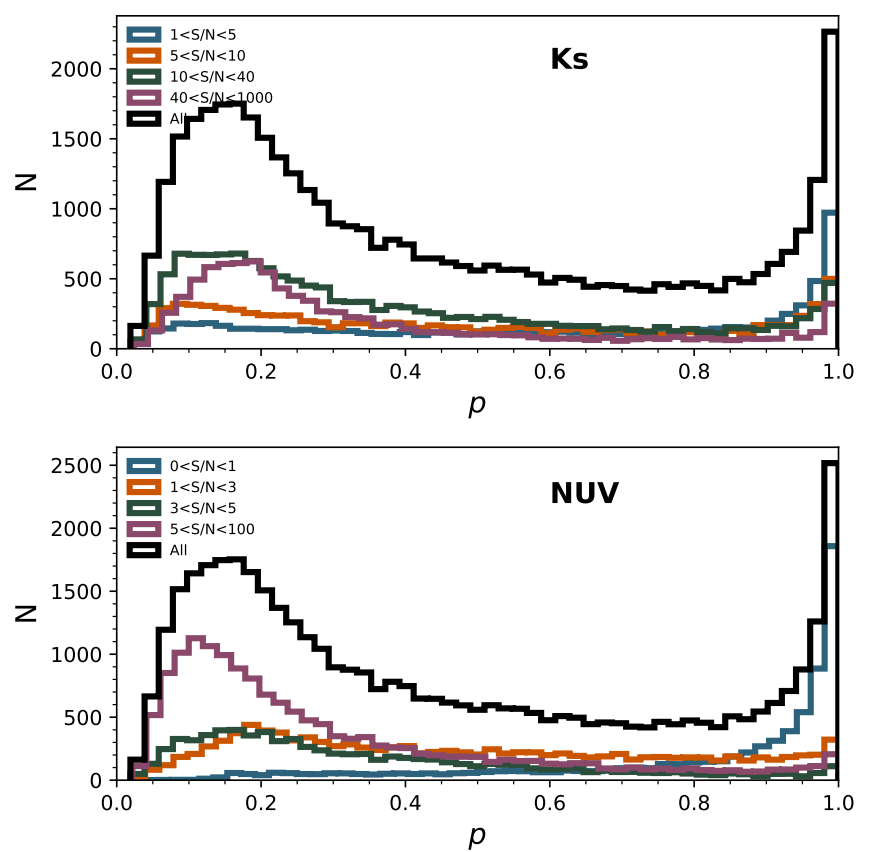

Fig. A.1. Distribution of the predictions $\hat{p}\left(m=1 \mid x_{\mathrm{obs}}\right)$ as a function of $K s$ band S/N (top panel) and NUV S/N (bottom panel). The different colors are for different selections in $\mathrm{S} / \mathrm{N}$ in each panel.

\section{Appendix B: Bayesian evidence}

The evidence $p\left(x_{\mathrm{obs}} \mid m\right)$ is a normalized probability density that represents the distribution of datasets drawn from the $m$ th model, regardless of the value of the parameter $\theta_{m}$ from its prior distribution. If models $m=0$ and $m=1$ are nested, the region of the data space of non-negligible probability under model $m=0$ has also a non-negligible probability under model $m=1$. Moreover, because model $m=1$ can fit to many more datasets, the probability density $p\left(x_{\mathrm{obs}} \mid m=1\right)$ is much more diffuse than the density $p\left(x_{\mathrm{obs}} \mid m=0\right)$. We therefore expect for datasets $x$ that can be explained by both models $m=0,1$ that $p(x \mid m=1) \leq$ $p(x \mid m=0)$. If the prior probabilities $p(m=0)$ and $p(m=1)$ of both models are equal, it implies that for datasets $x_{\mathrm{obs}}$ that can be explained by both models, $p(m=1 \mid x) \leq p(m=0 \mid x)$.

\section{Appendix C: Parameter tuning for classification methods}

The training catalog was used to optimize the value of $\phi$ with a specific algorithm given $\psi$, and the validation catalog was used to fit the tuning parameters $\psi$. To fit $\phi$ to a catalog of simulated datasets $\left(m^{i}, x^{i}\right), i \in I$, the optimization algorithm specified with the machine-learning model maximizes

$$
\prod_{i \in I} L\left(\hat{p}\left(m=1 \mid x^{i}\right) ; m^{i}\right), \text { where } L(p ; m)= \begin{cases}p & \text { if } m=1 \\ (1-p) & \text { if } m=0\end{cases}
$$

given the value of $\psi$. Generally, this optimization algorithm was run for several values of $\psi$. Then, the validation catalog was used to calibrate the tuning parameters $\psi$ based on data: the accuracy of $\hat{p}_{\psi}(m=1 \mid x)$ for many possible values of $\psi$ was computed on the validation catalog, and we selected the value $\hat{\psi}$ that led to the best results on this catalog. The resulting output of this twostep procedure is the approximation $\hat{p}_{\hat{\psi}}(m \mid x)$, which can easily be evaluated for the new dataset $x^{\prime}$. The accuracy of $\hat{p}(m=1 \mid x)$ can be measured with various metrics. The most common metric is the classification error rate on a catalog of $\left(m^{j}, S\left(x^{j}\right)\right), j \in J$, of $|J|$ simulations. We relied on this metric. It is is defined by the frequency at which the datasets $x^{j}$ are not well classified, that is,

$\frac{1}{|J|} \sum_{j \in J} \mathbf{1}\left\{\hat{m}^{j} \neq m^{j}\right\}$, where $\hat{m}^{j}= \begin{cases}1 & \text { if } \hat{p}\left(m=1 \mid x^{j}\right)>1 / 2 \\ 0 & \text { if } \hat{p}\left(m=1 \mid x^{j}\right) \leq 1 / 2\end{cases}$ 